\title{
Gail Model Underestimates Breast Cancer Risk in Thai Population
}

\author{
Doonyapat Sa-Nguanraksa ${ }^{1}$, Thanyawat Sasanakietkul ${ }^{1,2}$, Chayanuch \\ O-Charoenrat ${ }^{1}$, Anchalee Kulprom ${ }^{1}$, Pornchai O-Charoenrat ${ }^{1 *}$
}

\begin{abstract}
Background: The Gail model is the most widely used method for breast cancer risk estimation. This model has been studied and verified for its validity in many groups but there has yet to be a study to validate the Gail model in a Thai population. This study aims to evaluate whether the Gail model can accurately calculate the risk of breast cancer among Thai women. Methods: The subjects were recruited from the Division of Head, Neck, and Breast Surgery, Department of Surgery, Siriraj Hospital. The patients attending the division were asked to enroll in the study and complete questionnaires. Gail model scores were then calculated. Relationships between parameters were examined using the Pearson's chi-square test, Fisher's exact test, and independent-samples t-test. Results: There were 514 women recruited. Age, parity, age at first-live birth, and history of atypical ductal hyperplasia (ADH) were significant risk factors for breast cancer. The 5-year and lifetime risk score for breast cancer calculated by the Gail model were not significantly different between the patient and the control subjects. The proportions of the subjects with lifetime risk $\geq 20 \%$ were significantly higher in breast cancer patients $(p=0.049)$. Conclusion: The Gail model underestimated the risk of breast cancer in Thai women. Calibration of the model is still required before adoption in Thai population.
\end{abstract}

Keywords: Atypical hyperplasia- breast cancer- Gail model- risk assessment- Thai

Asian Pac J Cancer Prev, 20 (8), 2385-2389

\section{Introduction}

The incidence of breast cancer in Thailand is increasing. From 2010-2012, the mean annual age-standardized incidence rate of female breast cancer per 100,000 was 28.5 (Imsamran et al., 2015). The incidence was approximately 3 -times higher in Western Europe (96 per 100,000) (Ferlay et al., 2015). This difference may be due to differences in lifestyle, ethnicity, environmental factors, or socio-economic status.

The Breast Cancer Risk Assessment Tool is based on a statistical model known as the "Gail model" which is named after Dr. Mitchell Gail, Senior Investigator in the Biostatistics Branch of the National Cancer Institute's Division of Cancer Epidemiology and Genetics. The original Gail model used data from the Breast Cancer Detection Demonstration Project (BCDDP) and included five factors: age, age at first menstruation, age at first child, number of breast biopsies, and familial history of breast cancer in 1st degree relatives (Gail et al., 1989). The calculated result provides the risks of invasive and in situ carcinoma. The National Surgical Adjuvant Breast and Bowel Project (NSABP) modified this model by excluding the incidence of ductal carcinoma in situ
(DCIS) and including the risk factor of having atypical ductal hyperplasia (ADH). This modified Gail model has been validated using independent data in a study of 5,969 American white women aged 35-years-old or above (Costantino et al., 1999). The average follow-up period was 48.4 months and the overall ratio of expected to observed (E/O) breast cancer cases was 1.03 for women in the control arm of the Breast Cancer Prevention Trial. It has been concluded that the Gail model is well-calibrated, thus, the modified Gail model was used as the area of interest in this study.

In the calculations of the Gail model, several risk factors for breast cancer were not included. These include: history of breast biopsies that reveal lobular carcinoma in situ (LCIS); atypical lobular hyperplasia (ALH); ADH; previous radiation therapy to the chest wall; history of mutation in either the BRCA1 or BRCA; or a diagnosis of a genetic syndrome that may be associated with an elevated risk of breast cancer. Considering the lower incidence of breast cancer in Thailand compared to that of Western Europe, the Gail model that was formulated based on a Caucasian population may not accurately estimate the risk of breast cancer in a Thai sample. In this study, we attempted to validate the accuracy of the Gail model 
as well as evaluate several factors that may increase the risk of breast cancer, including body-mass index (BMI) and history of hormonal treatment or contraceptive drugs.

\section{Materials and Methods}

\section{Patients}

Breast cancer patients and control subjects were enrolled from January 2013 to December 2014 at the Division of Head, Neck, and Breast Surgery, Department of Surgery, Faculty of Medicine, Siriraj Hospital. Patients with invasive breast carcinoma aged 35-years-old or older were assigned to the case group. Thai women within the same age group who underwent mammography screening and breast ultrasonography which revealed BIRADS I or II and had no history of previous breast cancer or irradiation to their chest wall were assigned to the control group. All subjects completed the given questionnaires. Information regarding age, body mass index (BMI), family history of breast cancer, age at first live birth, parity, age at first menses, age of menopause, history of hormonal usage, and previous history of breast biopsy were recorded. This study was approved by Siriraj Institutional Review Board (certificate of approval number Si432/2014).

\section{Breast cancer risk assessment}

The Gail model risk score was calculated using available SAS codes for GM prediction from the NCI's Breast Cancer Risk Assessment Tool website (NCI Breast Cancer Risk Assessment Tool, available at http://www. cancer.gov/bcrisktool/Default.aspx). After consolidating data from both groups, the five-year risk score and lifetime risk score for each person was retrieved. The five-year risk score is the probability of patients developing breast cancer in the next five years and the lifetime risk score is the chance of developing breast cancer up to the age of 90 . The number of patients who had a five-year risk score of $>1.7 \%$ and a lifetime risk score of $>20 \%$ were recorded as these patients have an elevated breast cancer risk.

\section{Statistical analysis}

Data was analyzed by SPSS for Windows, version 21.0 (SPSS, IBM, Ehningen, Germany). Relationships between parameters and breast cancer risk were examined using the Pearson's chi-square test and the Fisher's exact probability test for dichotomized variables. Independentsamples t-test was used for continuous variables. The factors that had $\mathrm{p}$-value of $<0.1$ were entered in binary logistic regression to exclude the confounding factors. $\mathrm{P}$-values of $<0.05$ were considered statistically significant.

\section{Results}

\section{Characteristics of the enrolled subjects}

There were 514 subjects enrolled in this study: 251 breast cancer patients and 263 controls. The characteristics of the subjects are summarized in Table 1. The breast cancer group had a significantly greater average age, higher parity, were of an older age at first live birth, and had a more frequent history of ADH. Multivariate analysis by binary logistic regression showed that age and history of ADH were the independent risk factors for breast cancer ( $<<0.001$ and $\mathrm{p}=0.006$, respectively).

\section{Breast cancer risk assessment}

The mean 5-year risk score and lifetime risk score for

Table 1. Characteristics of the Enrolled Subjects

\begin{tabular}{|c|c|c|c|c|}
\hline & $\begin{array}{l}\text { Control, } \mathrm{n}=263 \\
\text { mean } \pm \mathrm{SD} / \mathrm{n}(\%)\end{array}$ & $\begin{array}{c}\text { Breast cancer, } \mathrm{n}=251 \\
\text { mean } \pm \mathrm{SD} / \mathrm{n}(\%)\end{array}$ & $\begin{array}{l}\text { Mean difference/Odds } \\
\text { ratios }(95 \% \mathrm{CI})\end{array}$ & $p$-value \\
\hline Age (years) & $54.08 \pm 8.38$ & $57.44 \pm 9.69$ & $3.36(1.79-4.93)$ & $<0.001$ \\
\hline $\operatorname{BMI}\left(\mathrm{km} / \mathrm{m}^{2}\right)$ & $24.40 \pm 4.09$ & $24.68 \pm 4.28$ & $0.28(-0.45-1.00)$ & 0.456 \\
\hline Age at menarche (years) & $14.14 \pm 2.11$ & $14.25 \pm 1.89$ & $0.11(-0.24-0.46)$ & 0.534 \\
\hline \multicolumn{5}{|l|}{ Parity } \\
\hline Nulliparous & $97(36.9)$ & $71(28.3)$ & 1 (reference) & \\
\hline Parous & $166(63.1)$ & $180(71.7)$ & $1.48(1.02-2.15)$ & 0.038 \\
\hline Age at first live birth (years) & $18.45 \pm 12.84$ & $21.10 \pm 12.39$ & $2.65(0.36-4.95)$ & 0.023 \\
\hline \multicolumn{5}{|l|}{ Age at first live birth group } \\
\hline$\leq 20$ & $28(16.9)$ & $27(15.0)$ & 1 (reference) & \\
\hline$>20-\leq 35$ & $132(79.5)$ & $137(76.1)$ & $1.08(0.60-1.92)$ & 0.804 \\
\hline$>35$ & $6(3.6)$ & $16(8.9)$ & $2.73(0.94-8.63)$ & 0.059 \\
\hline Duration of hormonal usage (years) & $1.86 \pm 3.61$ & $2.27 \pm 4.13$ & $0.41(-0.26-1.09)$ & 0.227 \\
\hline \multicolumn{5}{|c|}{ History of breast cancer in 1 st degree relative, $\mathrm{n}(\%)$} \\
\hline Absence & $218(82.9)$ & $208(82.9)$ & 1 (reference) & \\
\hline Presence & $45(17.1)$ & $43(17.1)$ & $0.99(0.63-1.57)$ & 0.979 \\
\hline Number of previous biopsies & $0.33 \pm 0.61$ & $0.30 \pm 0.63$ & $-0.04(-0.14-0.07)$ & 0.514 \\
\hline \multicolumn{5}{|l|}{ History of ADH } \\
\hline Absence & $262(99.6)$ & $235(93.6)$ & 1 (reference) & \\
\hline Presence & $1(0.4)$ & $16(6.4)$ & $8.89(2.02-39.05)$ & $<0.001$ \\
\hline
\end{tabular}


Table 2. Breast Cancer Risk Score of the Enrolled Subjects

\begin{tabular}{lcccc}
\hline & $\begin{array}{c}\text { Control, } \mathrm{n}=263 \\
\text { mean } \pm \mathrm{SD} / \mathrm{n}(\%)\end{array}$ & $\begin{array}{c}\text { Breast cancer, } \mathrm{n}=251 \\
\text { mean } \pm \mathrm{SD} / \mathrm{n}(\%)\end{array}$ & Mean difference (95\% CI) & $p$-value \\
\hline 5-year risk score & $0.802 \pm 0.487$ & $0.871 \pm 0.742$ & $-0.069(-0.178-0.040)$ & 0.218 \\
Lifetime risk score & $4.700 \pm 2.89$ & $5.287 \pm 4.315$ & $-0.586(-1.226-0.053)$ & 0.072 \\
5 -year risk $\geq 1.7, \%$ & $16(6.1)$ & $26(10.4)$ & & 0.077 \\
Lifetime risk $\geq 20, \%$ & $1(0.4)$ & $6(2.4)$ & & 0.049 \\
\hline
\end{tabular}

breast cancer and control subjects were not significantly different ( $\mathrm{p}=0.218$ and 0.072 , respectively). In the subjects who had a five-year risk score of $\geq 1.7 \%$, there was no statistically significant difference in the distribution between both groups $(\mathrm{p}=0.077)$. In the subjects who had a lifetime risk score of $\geq 20 \%$, there was a statistically significant difference in the risk between breast cancer patients and control subjects $(\mathrm{p}=0.049)$ (Table 2).

\section{Discussion}

Several breast cancer risk assessment models have been developed and applied mainly in Western populations (Claus et al., 1991; Parmigiani et al., 1998; Costantino et al., 1999; Amir et al., 2003). The Gail model, one of the most commonly applied models, has been previously used to calculate the risk for White, Black, and African-American women in Western countries (Gail et al., 1989; Adams-Campbell et al., 2007; Gail et al., 2007). Relatively few publications have evaluated the use of the Gail model in Asian populations and the reliability of this model has not been evaluated in a Thai population. Additionally, there is no standard risk assessment model for breast cancer in Thailand. The aim of this study was to determine risk factors for breast cancer and evaluate the Gail model using the database of a single major tertiary care center in Thailand.

We were able to identify risk factors for breast cancer such as older age, higher number of parities, older age at first live birth, and history of ADH. Most of these findings were concordant with previous reports (Kelsey and Bernstein, 1996; Winters et al., 2017). However, the control group had a higher proportion of nulliparous women. This finding was in contrast to several studies that report an association between being nulliparous and increased risk of breast cancer (Ma et al., 2006; Rojas and Stuckey, 2016). This disparity may be the result of a lower average age (under 20 years old) at which subjects in the control group had their first child compared to the breast cancer group. A shorter time between menarche and first live birth is associated with decreased breast cancer risk and this effect was more prominent in premenopausal women (Li et al., 2008). The lifetime risk of women who had children before 20 is $40 \%$ lower than that of women who had their first child aged 35 or older (Ewertz et al., 1990). Furthermore, the protective effect of pregnancy in decreasing risk of breast cancer development is counteracted by the adverse effect of the microenvironment of involuting mammary glands which may affect the patient for up to 5 years after delivery (Schedin et al., 2007).
The age at menarche and duration of hormonal usage were not different between the two groups. A possible hypothesis is that among the Thai population, the major factors that influence the development of breast cancer may not be directly related to hormonal changes. Similar data regarding the effect of the duration of estrogen exposure on the risk of breast cancer has been reported in a ten-year cohort study. In this study, 11,414 Thai women demonstrated no association between hormonal use and breast cancer risk (Poosari et al., 2014). In a Japanese population, analysis of 5,681 women also revealed that hormone-replacement therapy did not increase the incidence of breast cancer (Saeki et al., 2008).

In contrast to this, there appears to be a correlation between hormone-replacement therapy and an increased risk of breast cancer in Western populations. The cross-sectional age specific breast cancer incidence rate of patients older than 50 years was higher in the American Caucasian sample in comparison to the Asian-American sample. However, when analyzed by age group, the incidence of breast cancer amongst patients older than 50 years is increasing among the Asian population (Sung et al., 2015). As opposed to this, the incidence of breast cancer in American women aged 50 years or older has been on the decline since 2003, following the decrease in use of hormone-replacement therapy (Ravdin et al., 2007). Furthermore, across generations, breast cancer incidence is increasing among Asian-American women. Those who live in urban areas appear to have a higher risk than those who live in rural areas (Ziegler et al., 1993). In Thailand, premenopausal women usually have a short period of exposure to hormonal contraceptive pills and postmenopausal women do not commonly use hormonereplacement therapy. These examples seem to support the notion that environmental factors and lifestyle changes may impact Asian women's susceptibility to breast cancer, rather than the duration of their exposure to estrogen.

Family history of breast cancer in first degree relatives does not appear to have an association with breast cancer risk in this cross-sectional study. This finding may be due to a small sample size of subjects with a family history of breast cancer, and only $5 \%$ of all breast cancer cases arise from hereditary mutations in specific genes including BRCA1, BRAC2, and TP53 (Peto et al., 1999; Bakhuizen et al., 2019).

Among several factors identified in this study, $\mathrm{ADH}$ appears to be the strongest risk factor. Approximately 20\% of patients with ADH diagnosed by core needle biopsy had upstaging to breast cancer after surgical excision and more than $10 \%$ of patients with ADH developed cancer upon follow-up (Renshaw and Gould, 2016; Chiramongkol et 
al., 2017). Furthermore, the rate of cancer development was higher in atypical lobular hyperplasia (ALH). We suggest annual breast cancer screenings for patients with a history of these atypical lesions and chemoprevention should be discussed with the patients.

Although several factors were different between the case and control groups, risk scores calculated by the Gail model did not vary. The Gail model may underestimate the risk of developing breast cancer in both the control group and the breast cancer group. This seems to suggest that this model may not be applicable or accurate for a Thai sample or population. Other breast cancer risk assessment studies also found that the Gail model may not be suitable for Asian populations. A study by Chay et al. on Singaporean women with a 10 -year follow up found that the number of expected invasive breast cancer cases based on the Gail model was 1.85 times higher than the actual number (Chay et al., 2012). The refined Gail model for a South East Asian population had a limited discrimination power of 0.6 (Gao et al., 2012). A validation study of the modified Gail model in a Korean population also found that the model did not perform well in an Asian population. The refined Gail model also had limited discrimination power (Park et al., 2013).

The lack of statistical difference between the five-year and lifetime risk scores between the patients and controls may suggest that the Gail model underestimates the risk of developing breast cancer in a Thai population. Although the more than $20 \%$ lifetime risk was significantly higher in the breast cancer group, the data collected suggests further research must be done to elucidate which risk factors are specifically influencing a Thai population's breast cancer risk. In these high-risk individuals, counselling regarding risk reduction therapy should be considered.

One limitation of this study is lack of generalizability. As a cross-sectional study with a limited number of subjects from a single institute, the enrollment of the control group may have been subjected to elements of selective bias. The control group was enrolled from the breast cancer center at Siriraj Hospital, all of whom were attending for a mammography screening and ultrasonography. The subjects may have an implicit higher concern about developing breast cancer than the general population or may represent a higher socioeconomic or educational status that understands and is able to afford frequent health check-ups. These conditions may not be representative of the general Thai population. Conducting a future study at a multicenter large cohort may be required to further investigate the risk factors and to construct a more effective risk assessment model for breast cancer within the Thai population.

\section{References}

Adams-Campbell LL, Makambi KH, Palmer JR, et al (2007). Diagnostic accuracy of the Gail model in the Black Women's Health Study. Breast J, 13, 332-6.

Amir E, Evans DG, Shenton A, et al (2003). Evaluation of breast cancer risk assessment packages in the family history evaluation and screening programme. $J$ Med Genet, 40, 807-14.
Bakhuizen JJ, Hogervorst FB, Velthuizen ME, et al (2019). TP53 germline mutation testing in early-onset breast cancer: findings from a nationwide cohort. Fam Cancer, 18, 273-80.

Chay WY, Ong WS, Tan PH, et al (2012). Validation of the Gail model for predicting individual breast cancer risk in a prospective nationwide study of 28,104 Singapore women. Breast Cancer Res, 14, R19.

Chiramongkol N, Sa-nguanraksa D, Samarnthai N, et al (2017). Predictive factors for upgrading to breast cancer of atypical ductal hyperplasia on core needle biopsy in Thai population. J Med Assoc Thai, 100, 54-60.

Claus EB, Risch N, Thompson WD (1991). Genetic analysis of breast cancer in the cancer and steroid hormone study. $\mathrm{Am}$ J Hum Genet, 48, 232-42.

Costantino JP, Gail MH, Pee D, et al (1999). Validation studies for models projecting the risk of invasive and total breast cancer incidence. J Natl Cancer Inst, 91, 1541-8.

Ewertz M, Duffy SW, Adami HO, et al (1990). Age at first birth, parity and risk of breast cancer: a meta-analysis of 8 studies from the Nordic countries. Int J Cancer, 46, 597-603.

Ferlay J, Soerjomataram I, Dikshit R, et al (2015). Cancer incidence and mortality worldwide: sources, methods and major patterns in GLOBOCAN 2012. Int J Cancer, 136, 359-86.

Gail MH, Brinton LA, Byar DP, et al (1989). Projecting individualized probabilities of developing breast cancer for white females who are being examined annually. J Natl Cancer Inst, 81, 1879-86.

Gail MH, Costantino JP, Pee D, et al (2007). Projecting individualized absolute invasive breast cancer risk in African American women. J Natl Cancer Inst, 99, 1782-92.

Gao F, Machin D, Chow KY, et al (2012). Assessing risk of breast cancer in an ethnically South-East Asia population (results of a multiple ethnic groups study). BMC Cancer, 12, 529.

Imsamran W, Chaiwerawattana A, Wiangnon S, et al (2015). Cancer in Thailand volume VIII, 2010-2012. National cancer institute.

Kelsey JL, Bernstein L (1996). Epidemiology and prevention of breast cancer. Annu Rev Public Health, 17, 47-67.

Li CI, Malone KE, Daling JR, et al (2008). Timing of menarche and first full-term birth in relation to breast cancer risk. $\mathrm{Am}$ J Epidemiol, 167, 230-9.

Ma H, Bernstein L, Pike MC, et al (2006). Reproductive factors and breast cancer risk according to joint estrogen and progesterone receptor status: a meta-analysis of epidemiological studies. Breast Cancer Res, 8, R43.

NCI Breast Cancer Risk Assessment Tool [Online]. Available: http://www.cancer.gov/bcrisktool/Default.aspx [Accessed 2/21/2019].

Park B, Ma SH, Shin A, et al (2013). Korean risk assessment model for breast cancer risk prediction. PLoS One, 8 , e76736.

Parmigiani G, Berry D, Aguilar O (1998). Determining carrier probabilities for breast cancer-susceptibility genes $B R C A 1$ and BRCA2. Am J Hum Genet, 62, 145-58.

Peto J, Collins N, Barfoot R, et al (1999). Prevalence of BRCA1 and $B R C A 2$ gene mutations in patients with early-onset breast cancer. J Natl Cancer Inst, 91, 943-9.

Poosari A, Promthet S, Kamsa-ard S, et al (2014). Hormonal contraceptive use and breast cancer in Thai women. J Epidemiol, 24, 216-20.

Ravdin PM, Cronin KA, Howlader N, et al (2007). The decrease in breast-cancer incidence in 2003 in the United States. N Engl J Med, 356, 1670-4.

Renshaw AA, Gould EW (2016). Long term clinical follow-up of atypical ductal hyperplasia and lobular carcinoma in situ in breast core needle biopsies. Pathology, 48, 25-9. 
Rojas K, Stuckey A (2016). Breast cancer epidemiology and risk factors. Clin Obstet Gynecol, 59, 651-72.

Saeki T, Sano M, Komoike Y, et al (2008). No increase of breast cancer incidence in Japanese women who received hormone replacement therapy: overview of a case-control study of breast cancer risk in Japan. Int J Clin Oncol, 13, 8-11.

Schedin P, O'Brien J, Rudolph M, et al (2007). Microenvironment of the involuting mammary gland mediates mammary cancer progression. J Mammary Gland Biol Neoplasia, 12, 71-82.

Sung H, Rosenberg PS, Chen WQ, et al (2015). Female breast cancer incidence among Asian and Western populations: more similar than expected. J Natl Cancer Inst, 107, djv107.

Winters S, Martin C, Murphy D, et al (2017). Breast cancer epidemiology, prevention, and screening. Prog Mol Biol Transl Sci, 151, 1-32.

Ziegler RG, Hoover RN, Pike MC, et al (1993). Migration patterns and breast cancer risk in Asian-American women. J Natl Cancer Inst, 85, 1819-27.

\section{(ब®}

This work is licensed under a Creative Commons AttributionNon Commercial 4.0 International License. 